\title{
THE SPECTRAL THEORY OF SELF-ADJOINT WIENER-HOPF OPERATORS ${ }^{1}$
}

\author{
BY JOEL DAVID PINCUS
}

Communicated by M. Kac, April 5, 1966

Introduction. This note will present a new method for the construction of solutions to the Wiener-Hopf equation

$$
L x(s) \equiv \int_{0}^{\infty} k(s-t) x(t) d t=\xi x(s), \quad 0 \leqq s \leqq \infty .
$$

However, in addition to presenting an alternate to the WienerHopf factorization method, we will construct results not obtainable by that method; namely a complete spectral representation of selfadjoint operators of the given form. That is, we will construct a direct integral Hilbert space $\mathfrak{F}^{*}$ which will be characterized in terms of an integer-valued Lebesgue measurable multiplicity function, $m(\xi)$, which we will exhibit explicitly, and an isometric mapping of the basic Hilbert space onto $\mathfrak{K}^{*}$ given explicitly by a sequence of $m(\xi)$ integral operators whose kernels are generalized eigenfunctions of $L$; furthermore we will exhibit the transformation inverse to $S$ explicitly as a sum of integral operators acting on the components of $\mathfrak{F C}^{*}$.

These results will be obtained through a simple reduction to the author's previous work on Barrier Related Spectral Problems [1], [2], [3], and we will not require, as it is customary in the standard Wiener-Hopf procedure that $k(t)=O\left(e^{-a|t|}\right)$ for some $a>0$ or that $k(t)$ is real and continuous except for a finite number of jumps [4]. ${ }^{2}$

Finally, after exhibiting our method, we will show for a particular standard example how the textbook solutions can be recovered from our formulation.

Assumptions and basic definition. We will require Hermiticity throughout; namely, $k(t)=k(-t)^{-}$, and, in addition, we require that $k(t)$ and $|k(t)|^{2}$ be absolutely integrable. For simplicity, we also require that $\hat{k}(\lambda)$, the Fourier transform of $k(t)$, be nonnegative.

Reduction to canonical form. Let $P$ be the orthogonal projection from $L_{2}(-\infty, \infty)$ onto $L_{2}(0, \infty)$ such that

1 This work was performed under the auspices of the U. S. Atomic Energy Commission.

${ }^{2}$ See [5] however. 


$$
P x(t)= \begin{cases}x(t), & 0 \leqq t<\infty, \\ 0, & -\infty<t \leqq 0 .\end{cases}
$$

Let us consider the Fourier Transform, $\mathfrak{F}$, of the expression $L x(s)$ $=P \int_{0}^{\infty} k(s-t) x(t) d t$.

LEMMA.

$$
\mathfrak{F} P \mathfrak{F}^{-1} y(\lambda)=\frac{1}{2} y(\lambda)+\frac{1}{2 \pi i} \mathrm{P} \int_{-\infty}^{\infty} \frac{y(\mu)}{\mu-\lambda} d \mu .
$$

Proof. By the Paley-Wiener theorem $\mathfrak{F} P x$ is the limit in mean and almost everywhere of an analytic function $\Phi(z)$ such that $\int_{-\infty}^{\infty}|\Phi(x+y)|^{2} d x<$ constant, $0<y<\infty$. Furthermore, we may express $\Phi(z)$ in terms of its boundary values as:

$$
\Phi(z)=\frac{1}{2 \pi i} \int_{-\infty}^{\infty} \frac{\mathfrak{F} P x(\mu)}{\mu-z} d \mu .
$$

But the Plemelj [1] formulae tell us that

$$
\begin{aligned}
& \Phi(\lambda+i o)-\Phi(\lambda-i o)=\mathfrak{F} P x(\lambda) \\
& \Phi(\lambda+i o)-\Phi(\lambda-i o)=\frac{1}{\pi i} \mathrm{P} \int_{-\infty}^{\infty} \frac{\mathfrak{F} P x(\mu)}{\mu-\lambda} d \mu
\end{aligned}
$$

from which the desired conclusion follows.

It is now clear that

LEMMA.

$$
\hat{L} y(\lambda) \equiv \mathfrak{F} L \mathfrak{F}^{-1} y(\lambda)=\frac{1}{2} \hat{k}(\lambda) y(\lambda)+\frac{1}{2 \pi i} \mathrm{P} \int_{-\infty}^{\infty} \frac{\hat{k}(\mu) y(\mu)}{\mu-\lambda} d \mu .
$$

$\hat{L}$ is now in the form of a special example out of a class of operators for which the author was able to develop a complete spectral theory [2], [3].

Let $H$ be the Hilbert space which arises by completion out of the continuous functions on $(-\infty, \infty)$ with respect to the norm derived from the scalar product

$$
\langle f, g\rangle \equiv \int_{-\infty}^{\infty} \hat{k}(\mu) \mathfrak{F} f(\mu)(\mathfrak{F} g(\mu))-d \mu \equiv(\hat{k}(\cdot) \mathfrak{F} f(\cdot), \mathfrak{F} g(\cdot)) .
$$

LEммA. The operator $L$ on $H$ defined by setting $L x(s)$ $=\mathrm{P} \int_{u}^{\infty} k(s-t) x(t) d t$ is self-adjoint on $H$.

Proof. $\langle y, L x\rangle=\left(\hat{k} \mathfrak{F} y, \mathfrak{F} L \mathfrak{F}^{-1} \mathfrak{F} x\right)=(\hat{k} \mathfrak{F} y, \hat{L} \mathfrak{F} x)=\langle L y, x\rangle$.

LEMMA. Let $\hat{H}$ denote the completion in $H$ of the linear manifold of functions that vanish in $(-\infty, 0) . \hat{H}$ is annihilated by $L$. 
Proof. Let $x \in \hat{H} .\langle L x, y\rangle=\langle x, L y\rangle=0, \forall y$, because the range of $L$ is $\hat{H}$.

LEMMA. $L$ restricted to $\hat{H}$ has an absolutely continuous spectral measure.

Outline of Proof. Analyze $\hat{L}$ on the completion of $\mathfrak{F} L_{2}(0, \infty)$ in $L_{2}(-\infty, \infty ; \hat{k}(\mu) d \mu)$ through the approximation method given in (2) for the "splitting kernel" of singular integral operators. Specifically, consider the sequence of operators on $L_{2}\left(-\infty, \infty ; \hat{k}^{(n)}(\mu) d \mu\right)$ defined by setting

$$
\hat{L}^{(n)} x(\lambda)=\frac{1}{2} \hat{k}^{(n)}(\lambda) x(\lambda)+\frac{1}{2 \pi i} \mathrm{P} \int_{-\infty}^{\infty} \frac{\hat{k}^{(n)}(\mu)}{\mu-\lambda} x(\mu) d \mu
$$

where

$$
k^{(n)}(\lambda)= \begin{cases}k(\lambda), & -n \leqq \lambda \leqq n \\ 0, & \text { otherwise }\end{cases}
$$

REMARK. This construction shows that the only change which arises when the more general integral operators of (2) are studied on $(-\infty, \infty)$ rather than on a set of finite measure is that in the infinite case a trivial eigenmanifold of infinite multiplicity may exist. The absolutely continuous part of these operators is still given by the formulae developed in (2).

We turn to a construction of the generalized eigenfunctions of $L$.

Let

$$
E(l, z)=\exp \left\{\frac{1}{2 \pi i} \int_{-\infty}^{\infty} \int_{-\infty}^{\infty} g(\nu, \mu) \frac{d \nu}{\nu-l} \frac{d \mu}{\mu-z}\right\}
$$

where

$$
g(\nu, \mu)=\frac{1}{\pi} \arg \frac{\hat{k}(\mu)-\nu-i o}{-\nu-i o} .
$$

LEMMA. There exists a one-parameter family of positive purely singular measures of finite total mass, $d M_{\xi}()$, defined on the Borel sets of the real line and a positive function $A(\xi)$ such that

$$
\left(1-\exp \left\{-\int_{-\infty}^{\infty} g(\xi, \mu) \frac{d \mu}{\mu-z}\right\}\right)^{-1}=\int_{-\infty}^{\infty} \frac{d M_{\xi}(\mu)}{\mu-z}+A(\xi) z .
$$

Define $\left\{P_{\xi}^{(j)}(\mu)\right\}$ to be a complete orthonormal set in $L_{2}\left(d M_{\xi}(\mu)\right)$. 
Then let $F(\xi, z)=E(\xi+i o, z)-E(\xi-i o, z)$

$$
\begin{aligned}
& F_{1}(\xi, z) \equiv(E(\xi+i o, z)-E(\xi-i o, z))(A(\xi))^{1 / 2}, \\
& F_{j}(\xi, z) \equiv F(\xi, z) \int_{-\infty}^{\infty} P_{\xi}^{(j)}(\mu) \frac{d M_{\xi}(\mu)}{\mu-z}, \quad 1<j \leqq m(\xi)
\end{aligned}
$$

where $m(\xi)$ is the spectral multiplicity of $L$ and is computed as follows:

For each $\xi$ consider the set of $\mu$ 's for which $g(\xi, \mu)$ is different from zero. If this set is a union of $n$ disjoint intervals, then $m(\xi)=n$; otherwise, it is infinite. Let

$$
\begin{aligned}
& x_{1}(\xi, \lambda) \equiv \frac{1}{2 \pi i} \frac{F_{1}(\xi, \lambda+i o)-F_{1}(\xi, \lambda-i o)}{\hat{k}(\lambda)}, \\
& x_{j}(\xi, \lambda) \equiv \frac{1}{2 \pi i} \frac{F_{j}(\xi, \lambda+i o)-F_{j}(\xi, \lambda+i o)}{\hat{k}(\lambda)}, \quad 1<j \leqq m(\xi),
\end{aligned}
$$

and define a family of vector valued distributions mapping $H$ into $L_{2}(\sigma(L))$ by setting $\chi_{i}(\xi, \lambda)=\mathfrak{F}^{-1} x_{i}(\xi, \lambda)$; i.e.

$$
\left\langle f, \chi_{i}(\xi, \cdot)\right\rangle \equiv\left(\hat{k} \mathfrak{F} f, x_{i}(\xi, \cdot)\right)^{3} \text { for } f \in L_{2}(-\infty, \infty) .
$$

THEOREM. The distributions $\chi_{i}(\xi, \cdot)$ form a complete orthonormal set of generalized eigenfunctions for $L$ on $\hat{H}$. Thus, if we set $t^{4}$

$$
\begin{aligned}
\delta f & =\left(g_{1}(\xi), \cdots, g_{m(\xi)}(\xi)\right)=g(\xi) \text { with } g_{i}(\xi)=\left\langle f, \chi_{i}(\xi, \cdot)\right\rangle, \text { then } \\
s L f & =\xi g(\xi), \\
f(\lambda) & =\int_{\sigma(L)} \sum_{1}^{m(\xi)} \chi_{i}(\xi, \lambda) g_{i}(\xi) d \xi, \text { and } \\
\langle f, f\rangle & =\|f\|_{H}^{2}=\int_{\sigma(L)} \sum_{i=1}^{m(\xi)}\left|g_{i}(\xi)\right|^{2} d \xi .
\end{aligned}
$$

An Example. Consider the eigenvalue problem for the Lalesco Equation (4), (6)

$$
L x(\lambda)=\frac{(2 \pi)^{1 / 2}}{2} \int_{0}^{\infty} \exp (-|\mu-\lambda|) x(\mu) d \mu=\xi x(\lambda),
$$

3 The proof that this definition defines a continuous transformation is contained in the results of (1) and (2). In addition, an alternate characterization of these distributions as continuous linear functionals on a space of infinitely differentiable functions of rapid decrease at infinity is also discussed in (2).

4 This correspondence is first defined for $f \in L_{2}(-\infty, \infty)$. Then it is extended to $H$, using the boundedness implied by the isometry statement of the theorem. 


$$
\hat{k}(\lambda)=\frac{1}{(2 \pi)^{1 / 2}} \int_{-\infty}^{\infty} \exp (-|x|+i x \lambda) d x=\frac{1}{1+\lambda^{2}} .
$$

The standard Wiener-Hopf theory proceeds by factoring $1-(2 \pi)^{1 / 2} \hat{k}(\lambda)(7)$. Let us apply our formulae instead. After an integration by parts we may write

$$
\begin{aligned}
E(l, z)=\exp \left\{\frac { 1 } { 2 \pi i } \int _ { - \infty } ^ { \infty } \left[\frac{1}{u+(1 / l-1)^{1 / 2}}+\frac{1}{u-(1 / l-1)^{1 / 2}}\right.\right. \\
\left.\left.\quad-\frac{1}{u+i}-\frac{1}{u-i}\right] \ln (u-z) d u\right\} .
\end{aligned}
$$

Now taking $0<\operatorname{Re} l \leqq 1$ and $\operatorname{Im} l$ sufficiently small we can evaluate the above integral by residues, obtaining:

$$
\begin{array}{ll}
E(l, z)=\frac{-i-z}{-(1 / l-1)^{1 / 2}-z}, & \operatorname{Im} z>0, \quad \operatorname{Im} l>0, \\
E(l, z)=\frac{-i-z}{(1 / l-1)^{1 / 2}-z}, & \operatorname{Im} z>0, \quad \operatorname{Im} l<0, \\
E(l, z)=\frac{(1 / l-)^{1 / 2}-z}{i-z}, & \operatorname{Im} z<0, \quad \operatorname{Im} l>0, \\
E(l, z)=\frac{-(1 / l-1)^{1 / 2}-z}{i-z}, & \operatorname{Im} z<0, \quad \operatorname{Im} l<0,
\end{array}
$$

from which we obtain in turn:

$$
F(\xi, \lambda+i o)-F(\xi, \lambda-i o)=\frac{2(1 / \xi-1)^{1 / 2}}{1 / \xi-1-\lambda^{2}}(\lambda+i)-\frac{2(1 / \xi-1)^{1 / 2}}{i-\lambda}
$$

or

$$
\frac{F(\xi, \lambda+i o)-F(\xi, \lambda-i o)}{\hat{k}(\lambda)}=\frac{2}{\xi}\left(\frac{1}{\xi}-1\right)^{1 / 2} \frac{\lambda+i}{\frac{1}{\xi}-1-\lambda^{2}}
$$

which is, except for the normalization, the standard result for the (generalized) Fourier Transform of the eigendistribution of $L$ corresponding to the spectral point $\xi$. (The spectral multiplicity is of course identically equal to one in this example.)

Final remarks. The rather cumbersome proof given by the author in (2) can now be very much simplified by means of a new theory of singular-Riemann-Hilbert boundary value problems [3]. 


\section{REFERENCES}

1. J. D. Pincus, On the spectral theory of singular integral operators, Trans. Amer. Math. Soc. 113 (1964), 101-128.

2. - Commutators, generalized eigenfunction expansions, and singular integral operators, Trans. Amer. Math. Soc. 121 (1966), 358-377.

3. - A singular Riemann Hilbert problem, 1965 Summer Institute on Spectral Theory and Statistical Mechanics, Brookhaven National Laboratory, Upton, New York.

4. R. E. A. C. Paley and N. Wiener, Fourier transforms in the complex domain, Amer. Math. Soc. Colloq. Publ., Vol. 19, Amer. Math. Soc., Providence, R. I., 1934.

5. M. G. Kreĭn, Integral equations on a half-line, Amer. Math. Soc. Transl. (2) 22 (1962), 163-288.

6. E. C. Titchmarsh, Theory of the Fourier integral, Oxford University Press, Oxford, 1948.

Applied Mathematics Department

BRookhaven National Laboratory

Upton, LONG IsLAND, New YoRK 